\title{
A Comparative Study of Transcription Techniques for Nonlinear Optimal Control Problems Using a Pseudo-Spectral Method
}

\author{
Chang-Joo Kim* and Sangkyung Sung** \\ Department of Aerospace and Information Engineering, Konkuk University, Seoul 143-701, Korea
}

\begin{abstract}
This article investigates various transcription techniques for the Legendre pseudospectral (PS) method to compare the pros and cons of each approach. Eight combinations from four different types of collocation points and two discretization methods for dynamic constraints, which differentiate Legendre PS transcription techniques, are implemented to solve a carefully selected test set of nonlinear optimal control problems (NOCPs). The convergence property and prediction accuracy are compared to provide a useful guideline for selecting the best combination. The tested NOCPs consist of the minimum time, minimum energy, and problems with state and control constraints. Therefore, the results drawn from this comparative study apply to the solution of similar types of NOCPs and can mitigate much debate about the best combinations. Additionally, important findings from this study can be used to improve the numerical efficiency of the Legendre PS methods. Three PS applications to the aerospace engineering problems are demonstrated to prove this point.
\end{abstract}

Key words: Collocation Points, Gauss Quadrature, Nonlinear Optimal Control, Pseudospectral Transcription Techniques.

\section{Introduction}

The pseudospectral (PS) method is a direct collocation method, which generally outperforms indirect methods, owing to their large radii of convergence, and solves nonlinear optimal control problems (NOCPs) with any type of constraint [1-4]. In a PS method, the collocation points are specially designed to utilize the highly accurate quadrature formulas and the corresponding analysis converges at an exponential rate for problems with a smooth and well-behaved solution [5-7]. In this strategy, Legendre or Chebyshev interpolating polynomials are typically used to approximate states and controls in the continuous time domain. This paper focuses on a method based on Legendre polynomials for PS collocation. The set of collocation points, which are typically

used in the Legendre PS method, are classified as LegendreGauss (LG), Legendre-Gauss-Radau (LGR), flipped LegendreGauss-Radau (FLGR), and Legendre-Gauss-Lobatto (LGL) points, depending on how the end points are incorporated. Because the initial and final state conditions are generally imposed on an NOCP, the LGL points, which include two end points, are the most obvious choice. However, recent studies $[7,8]$ have revealed that the costate and control estimates using LGL points exhibit noisy and inaccurate behaviors. In contrast, Williams [9] successfully implemented LGL points via the integration method for a constrained Van der Pol oscillator problem, which demonstrated excellent agreement for both primal and dual solutions.

Another issue with the PS method is related to the methods of approximating the underlying dynamic equations. The integration method uses the quadrature formula after converting system dynamics into an integration form. Alternatively, the differentiation method directly applies a differention matrix to the derivative term of the state to obtain a system of nonlinear algebraic equations (NAEs). The differentiation method is generally preferred to the integration method beacuse the related Jacobian matrix is more sparse than in the integration method. However, differentiation methods still require a quadrature approximation to the system dynamics to predict the terminal states when the
This is an Open Access article distributed under the terms of the Creative Commons Attribution Non-Commercial License (http://creativecommons.org/licenses/by$\mathrm{nc} / 3.0 /$ which permits unrestricted non-commercial use, distribution, and reproduction in any medium, provided the original work is properly cited. (c) Associate Professor
** Associate Professor, corresponding author: sksung@konkuk.ac.kr 
final end point is not included in the collocation points as in the LG and LGR methods [6-8]. Also, convergence with the differentiation method is more sensitive to accuracy than the integration method in the initial estimates of the solution.

This paper investigates the effect of each selectable combination of transcription techniques on numerical convergence and solution accuracy of the Legendre PS method. Because the numerical characteristics of each approach are highly affected by the type of NOCP and by the constraints, we carefully selected six test problems with analytic solutions from various studies in the literature $[7,10-12]$ to encompass a wide range of NOCPs, such as the minimum time, minimum energy, and problems with or without state and control constraints. Convergence history and accuracy in predicting the states and controls for each problem are throughly investigated using the results with applicable combinations. Therefore, the results of this study can be used to identify the numerical advantages and disadvantages of each combination among transcription techniques, from which the best one can be selected considering each characteristics of the NOCP. Finally, various applications of the PS methods to the aircraft and spacecraft flight dynamics are demonstrated to show that the PS method can be a useful tool in solving complex aerospace engineering problems and in designing an optimal trajectory.

\section{Pseudospectral Transcription Techniques}

The NOCP suited to this study is represented by the standard Bolza form $[10,12]$ over a time interval of $t \in\left[t_{0}, t_{f}\right]$ for a general nonlinear dynamical system as

$$
\min J=\phi\left(\mathbf{x}_{f}, t_{f}\right)+\int_{t_{0}}^{t_{f}} F(\mathbf{x}, \mathbf{u}, t) d t
$$

subject to

$$
\begin{aligned}
& \dot{\mathbf{x}}=\mathbf{f}(\mathbf{x}, \mathbf{u}, t) \\
& g_{m}(\mathbf{x}, \mathbf{u}, t) \leq 0, \quad m=1, \cdots, m_{I} \\
& h_{n}\left(\mathbf{x}_{0}, \mathbf{x}_{f}, t_{0}, t_{f}\right)=0, \quad n=1, \cdots, n_{E}
\end{aligned}
$$

where $g_{m}, h_{n}, \mathrm{x}_{0}$, and $\mathrm{x}_{f}$ represent the path constraints, end constraints, initial states, and final states, respectively. Eqs (1)-(3) can be transcribed into a solvable nonlinear programming problem (NLP) using the Legendre PS method after applying the affine transformation $\tau=2\left(t-t_{\mathrm{o}}\right) /\left(t_{f}-t_{\mathrm{o}}\right)-1$ and using collocation points $\left\{\tau_{k}\right\}_{k=0}^{K}[5,6]$. For this purpose, the integration and differentiation for a function $f(\tau)$ are typically approximated using the PS transcription formulas as shown in (4)-(6).

$$
\begin{aligned}
& \int_{-1}^{1} f(\tau) d \tau \approx \sum_{j=0}^{K} w_{j} f\left(\tau_{j}\right) \\
& \int_{-1}^{\tau_{j}} f(\tau) d \tau \approx \sum_{j=0}^{K} I_{j, k} f\left(\tau_{k}\right) \\
& \frac{d f\left(\tau_{j}\right)}{d \tau} \approx \sum_{k=0}^{K} D_{j k} f\left(\tau_{k}\right)
\end{aligned}
$$

where $w_{f}, I_{j, k}$ and $D_{j, k}$ represent the quadrature weight, the elements in the integration and differentiation matrices, respectively.

The quadrature weights, the differentiation and integration matrices can be computed with extremely high accuracy using orthogonal basis functions. The Legendre PS methods utilize the Legendre polynomials as orthogonal basis functions, which are orthogonal to the unit weight function over the interval $\tau \in[-1,1]$. The quadrature formula is defined using the set of quadrature (or collocation) points and their corresponding weights. The most commonly used sets are LG, LGR, FLGR, and LGL points. The $(K+1)$ collocation points for each set are defined by the roots of the polynomial equations, as listed in Table Al in Appendix A. Fig. 1 shows the distribution of six points (with $K=5$ ) for each set of collocation points. The LGL points include both end points whereas the LG points do not include either end point. The FLR and FLGR points contain one of the end points. The weights $\left\{w_{k}\right\}_{k=0}^{k=K}$ are summarized in Table A2 for each set of collocation points. The computational domain typically includes both end points to impose the initial and final conditions. This domain can be divided by $(N+1)$ computational nodes $\left\{-1=\tau_{0}<\tau_{1}<\cdots<\tau_{N}=1\right\}$. A unified defintion for computational nodes and corresponding weights for a different set of the collocation points can be achieved using the formulas shown in Table A3. Also, the formulas required to compute the integration and differentiation matrices are contained in Appendix A.

The two different ways of transcribing the system dynamics are the integration method and the differentiation method. The integration method uses a partial integration form of the motion equations and approximates it using the integration formula as shown in (5). The result can be written

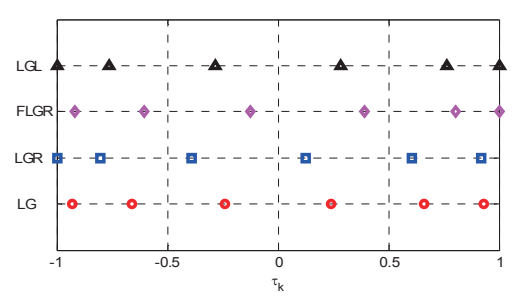

Fig. 1. Distribution of collocation points. 
for $j=0, \cdots, K$ as

$$
\mathbf{x}\left(\tau_{j}\right) \approx \mathbf{x}(-1)+\frac{t_{f}-t_{0}}{2} \sum_{k=0}^{K} I_{j k} \mathbf{f}\left(\mathbf{x}_{k}, \mathbf{u}_{k}, t_{k}\right)
$$

Whereas, the differentiation method directly applies (6) to the dynamic constraints in order to get the following algebraic constraints at each collocation point.

$$
\sum_{k=0}^{K} D_{j k} \mathbf{x}_{k}=\frac{t_{f}-t_{0}}{2} \mathbf{f}\left(\mathbf{x}_{j}, \mathbf{u}_{j}, t_{j}\right)
$$

Theobjectivefunction shownin(1)canbestraightforwardly approximated using (4) and the constraints defined by (3) can be satisfied at each of collocation points. As the results of the above approximations, the NOCP defined through (1)(3) can be transformed into a solvable NLP.

The NLP formulated both for the differentiation and integration methods are solved using the robust SQP (rSQP) algorithm proposed by Fabien [13]. The algorithmic details are illustrated in [14] with its applications to both the example NLP test problems and to the NOCP analyses. Another important point in selecting a transcription method is related to the prediction capability of the boundary control. If one of the end points is not included in the set of collocation points, as in LG, LGR, and FLGR methods, the boundary controls at such points cannot be predicted. Therefore, these controls should be estimated when required for applications. A simple extrapolation method or a rigorous prediction method using Pontryagin's minimum principle [6] can be utilized. However, this paper does not address this topic.

\section{Comparative Studies Using Nocp Test Problems}

The comparative studies on various collocation techniques are conducted with six test problems with analytic solutions, which are defined in Appendix B. Table 1 summarizes the character of each problem using the identification number (ID) and carefully selected studies from the literature $[7,10$ 12] to consider the problem-dependent properties of each method. If not specifically mentioned, the following analysis conditions are commonly applied to initialize solutions in a unified manner and to define the termination conditions of the iterative NLP process.

- For the problems with a free final time, the final time is initially set to seven seconds.

- When the final states are not prescribed, states are initialized with the initial states; otherwise, they are estimated using the linear interpolation.

- The initial estimate of control is set to zero. In a case when the control is constrained by both upper and lower limits, it is initialized with the averaged value.

- When the differentiation method is used, the differential approximation of system dynamics in (8) is applied with the modified differentiation matrix, if required, for all the collocation points.

- Analyses are conducted with 40 computational nodes and terminated when the maximum constraint violation is less than $10^{-6}$ and one of the following conditions is met.

i) The norm of the Lagrangian function gradient for the NLP is less than $10^{-4}$.

ii) The norm of the merit function gradient for the line search algorithm is less than $10^{-4}$.

The PS methods are implemented using the Fortran 95 and executed on the Intel Core i7 CUP 960. The maximum constraint violation $V_{C}$ used as one of the termination conditions is defined as follows:

$$
V_{C}=\max \left(\left|h_{n}\right|, \max \left(g_{m}, 0\right), \quad \begin{array}{c}
n=1, \cdots, n_{E} \\
m=1, \cdots, m_{I}
\end{array}\right)
$$

Figures 2-7 present the analysis results for each NOCP test problem (TP1-TP6). The convergence characteristics and the prediction accuracy are compared with respect to the varying transcription techniques. For all test problems, the

Table 1. Summary of NOCP test problems

\begin{tabular}{|l|l|}
\hline ID & Descriptions and sources \\
\hline TP1 $^{*}$ & minimum energy problem with bounded control and non-smooth [7] \\
\hline TP2 & minimum energy problem with path constraint and non-smooth solution and [10] \\
\hline TP3 $^{*}$ & minimum time problem with bounded control [11] \\
\hline TP4 $^{*}$ & Brachistochrone problem without path constraint [10] \\
\hline TP5 $^{*}$ & Brachistochrone problem with path constraint [10] \\
\hline TP6 $^{*}$ & Singular problem with bounded control [12] \\
\hline & (note: * denotes problems with free final time)
\end{tabular}


analyses converge to the analytical solution with the present initialization method, which proves that the Legendre PS method has a large radius of convergence. The integration method generally outperforms the differentiation method for most test problems except for the cases of TP2, in which no differerences are clearly discernible in convergence
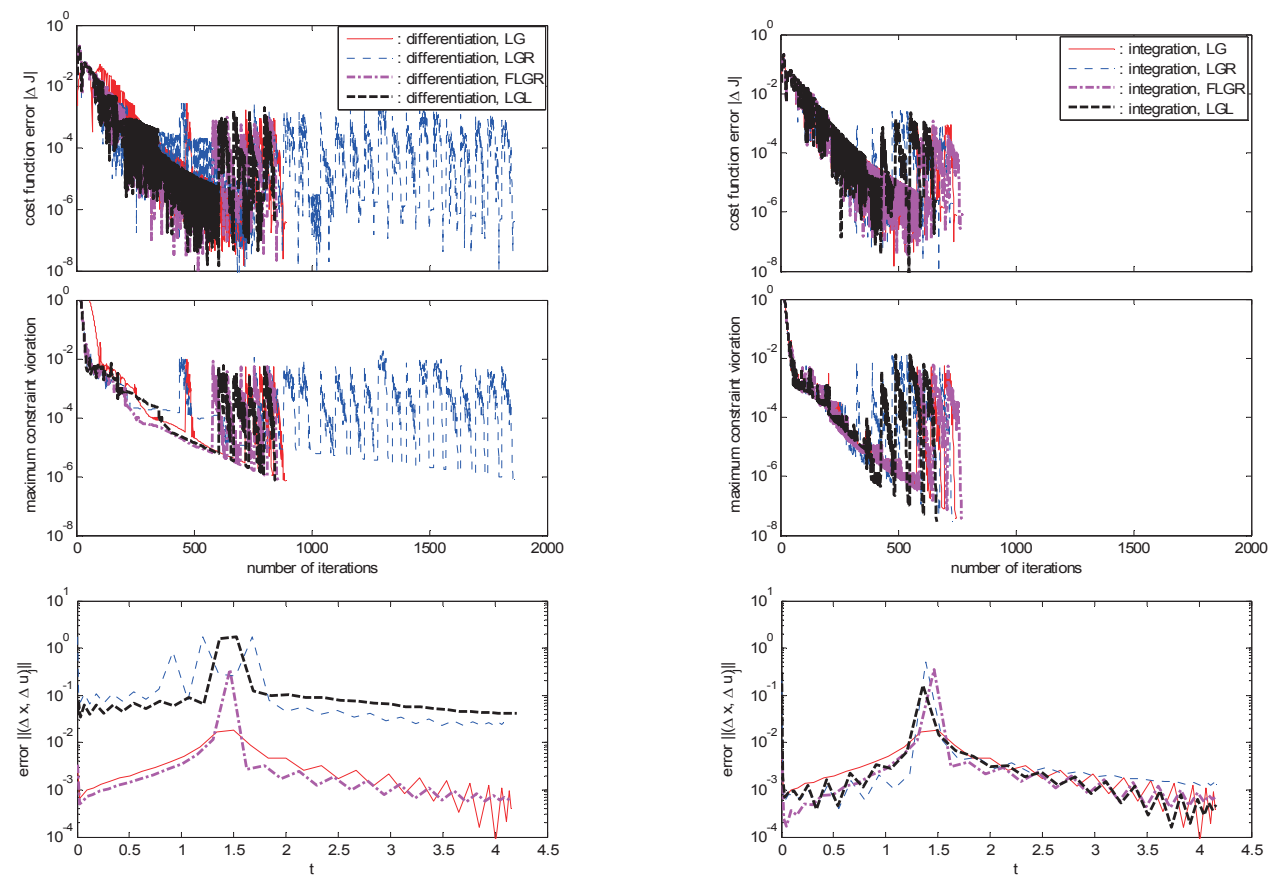

(a) Differentiation method

(b) Integration method

Fig. 2. Comparison of analysis results for TP1 (minimum energy problem with non-smooth solution)
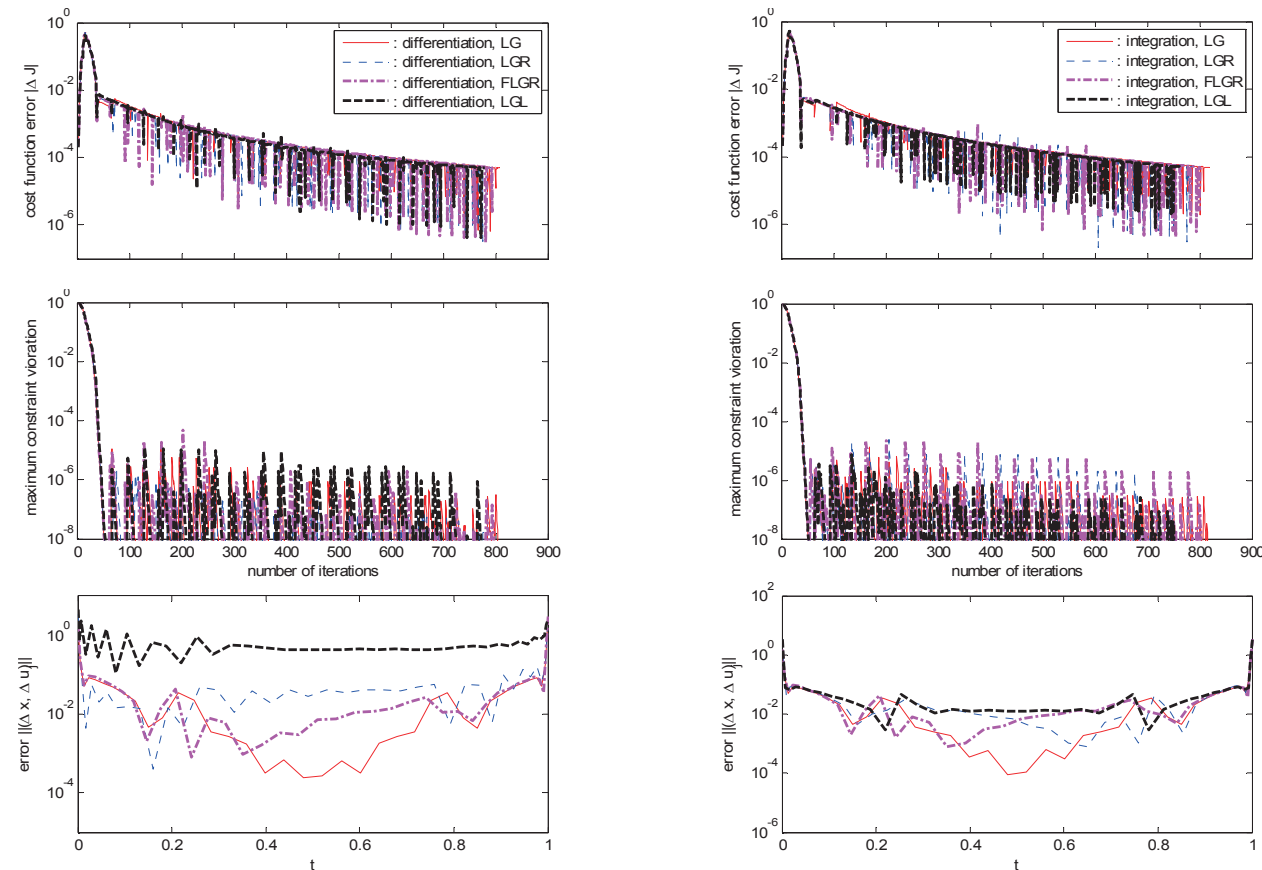

(a) Differentiation method

(b) Integration method

Fig. 3. Comparison of analysis results for TP2 (minimum energy problem with path constraint) 
characterics. The convergence and the accuracy of the difference method generally shows a higher dependency on the types of collocation points than those with the integration method. The integration method provides approximately the
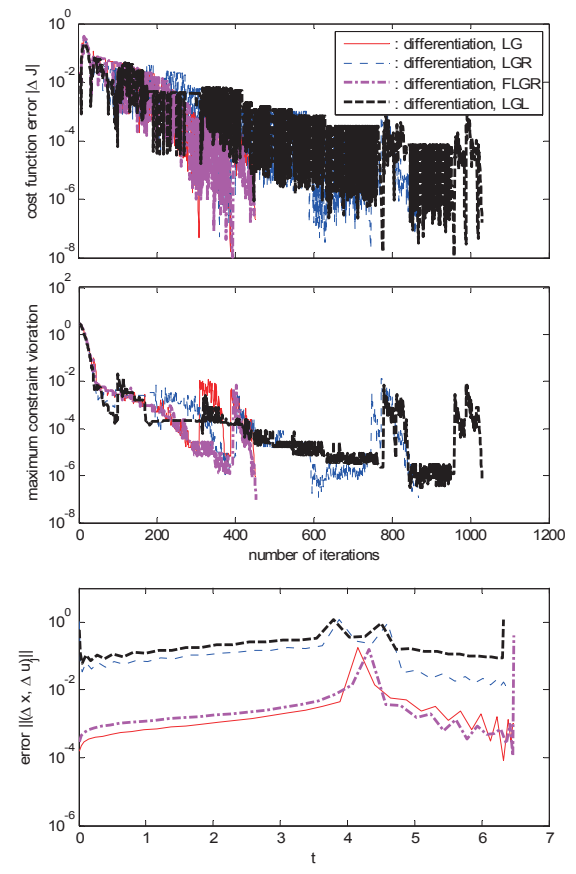

(a) Differentiation method same level of accuracy regradless of the types of collocation points, whereas the analyses using the differenciation method exhibit poor accuracy with LGR and LGL points compared to those with the LG and FLGR points.
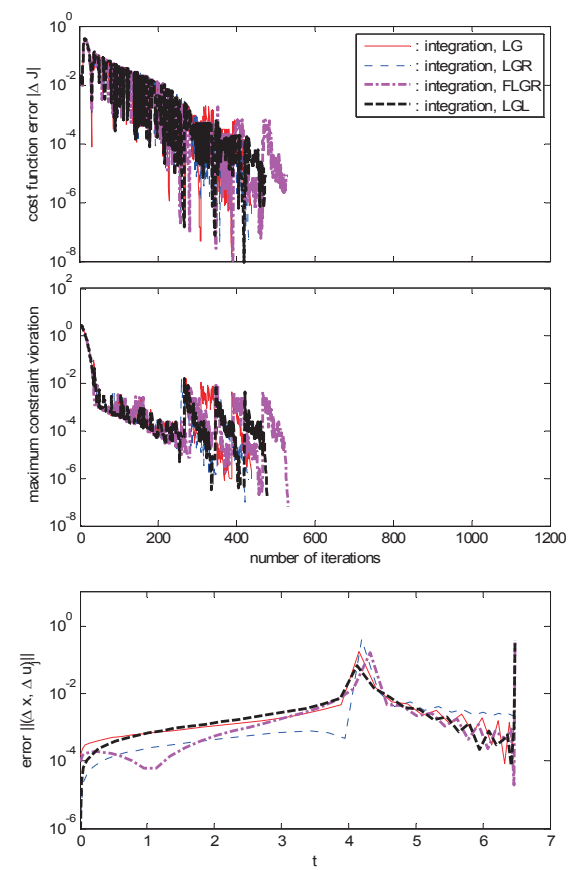

(b) Integration method

Fig. 4. Comparison of analysis results for TP3 (minimum time problem with bounded control)
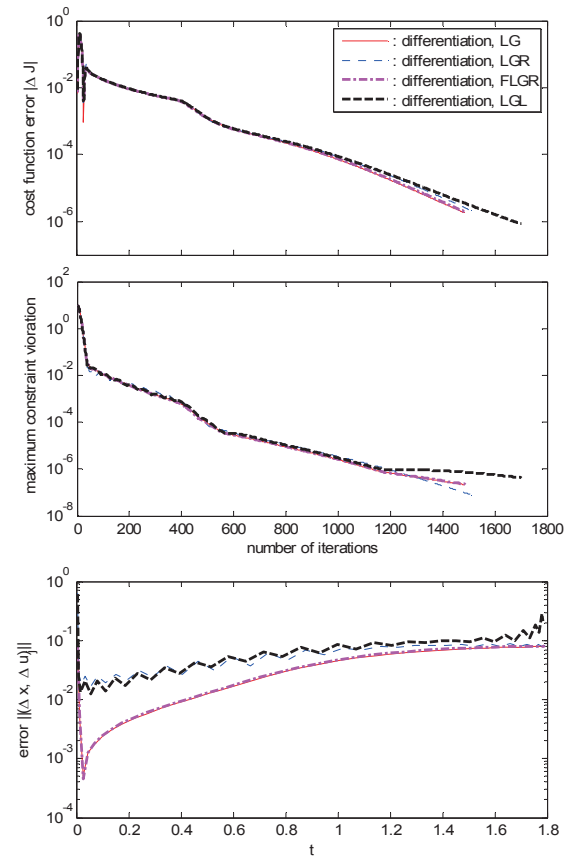

(a) Differentiation method
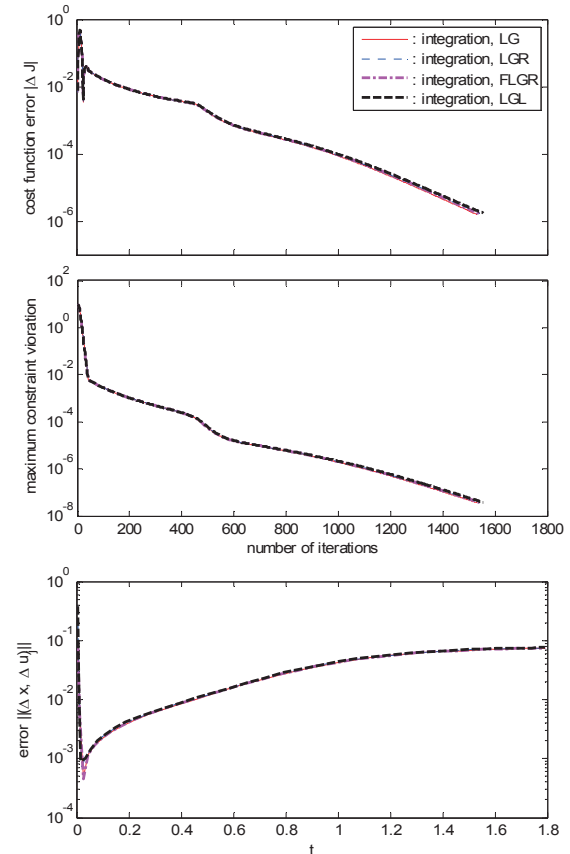

(b) Integration method

Fig. 5. Comparison of analysis results for TP4 (Brachistochrone problem without path constraint) 
The convergence characteristics and the prediction accuracy are indicated in the following figures. Fig. 8 demonstrates the norms of absolute errors in states and control predictions with respect to the number of iterations
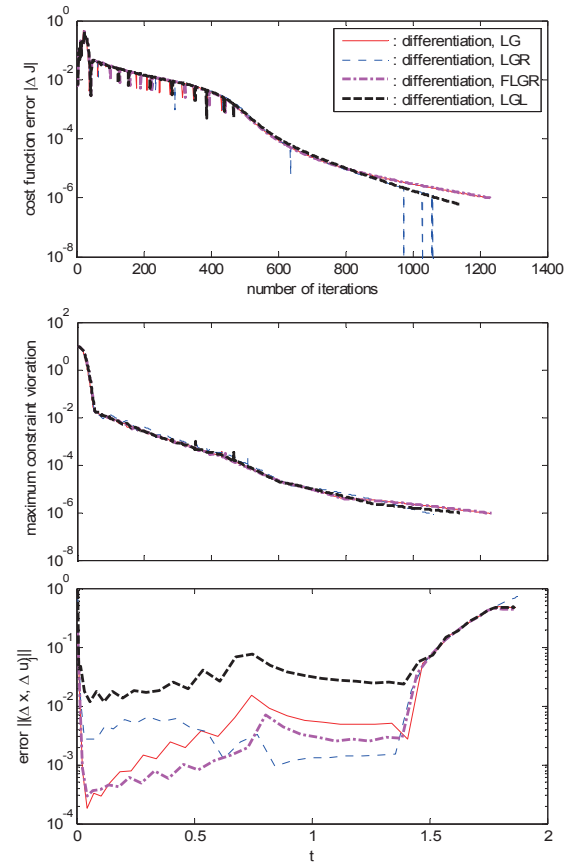

(a) Differentiation method for TP1 using the integration method and the LG points. The control estimate exhibits a relatively large error around the initial time and at the time with a control discontinuity. Because the control is constrained with upper and lower
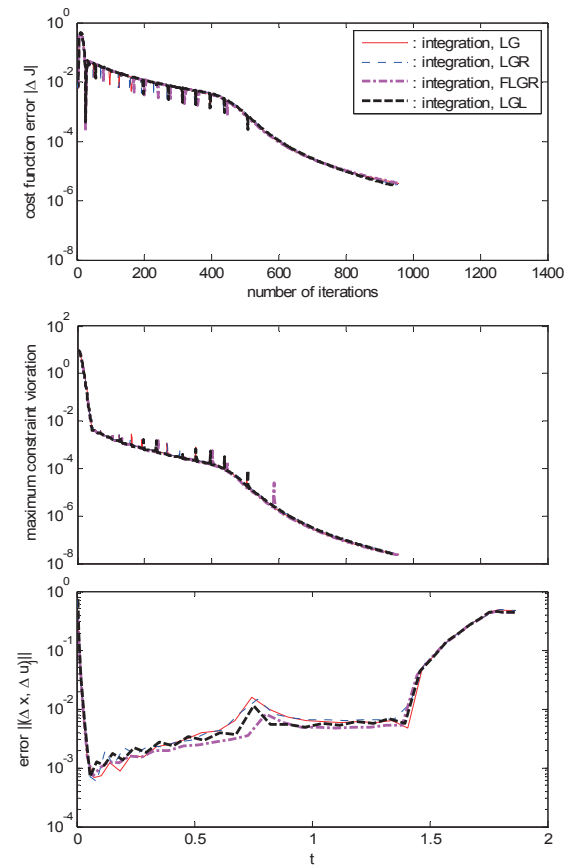

(b) Integration method

Fig. 6. Comparison of analysis results for TP5 (Brachistochrone problem with path constraint)
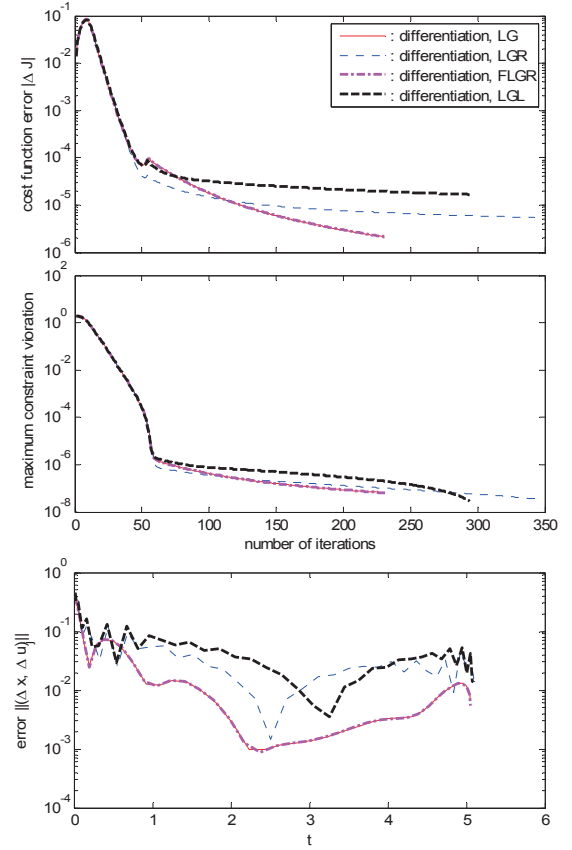

(a) Differentiation method
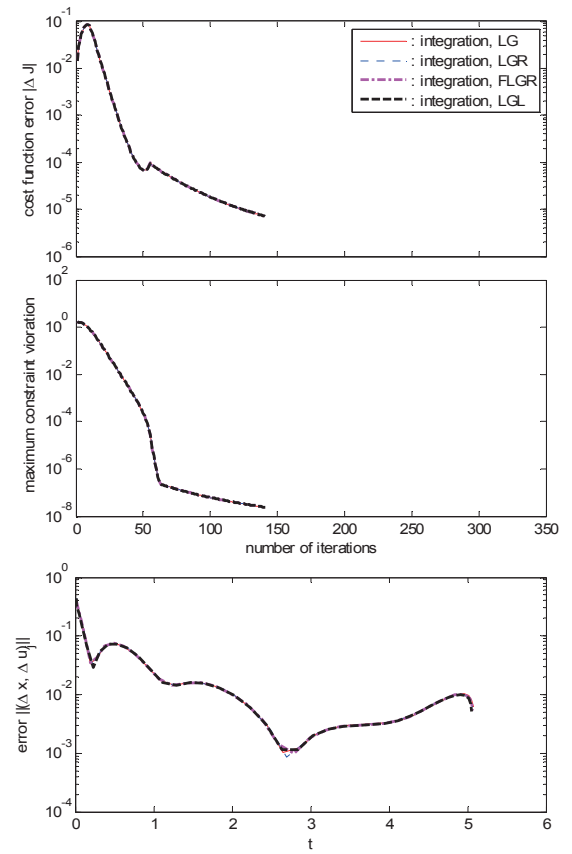

(b) Integration method

Fig. 7. Comparison of analysis results for TP6 (singular problem with path constraint) 
limits, the averaged value of 1.5 is used as the initial guess over all the computational nodes. The poor convergence around the initial time is related to the decrease in quadrature weights at collocation points near the initial time. This effect is also noted in Fig. 9 and Fig. 11 for TP2 and TP6, respectively. While the maximum error of the state prediction is decreased by two orders of magnitude compared to the first iteration, the control input still has a
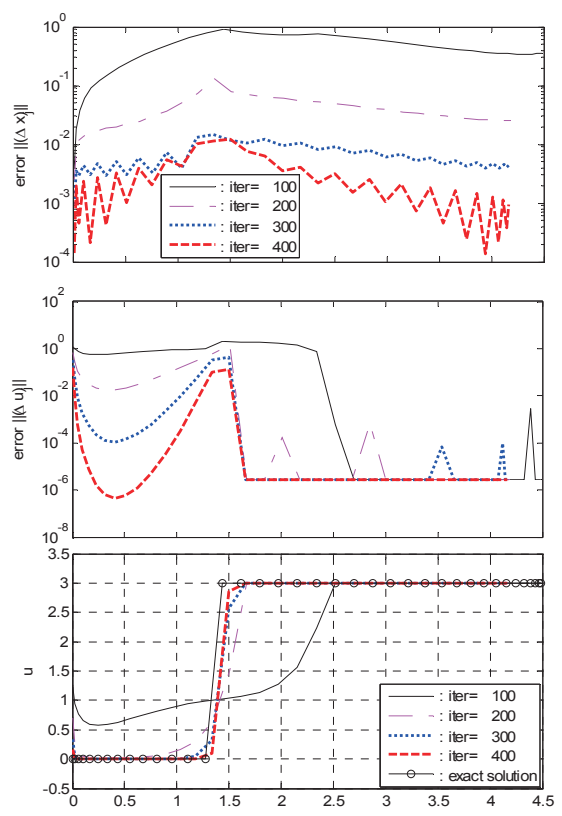

Fig. 8. Analysis results for LG points with integration method depending on number of iterations for TP1

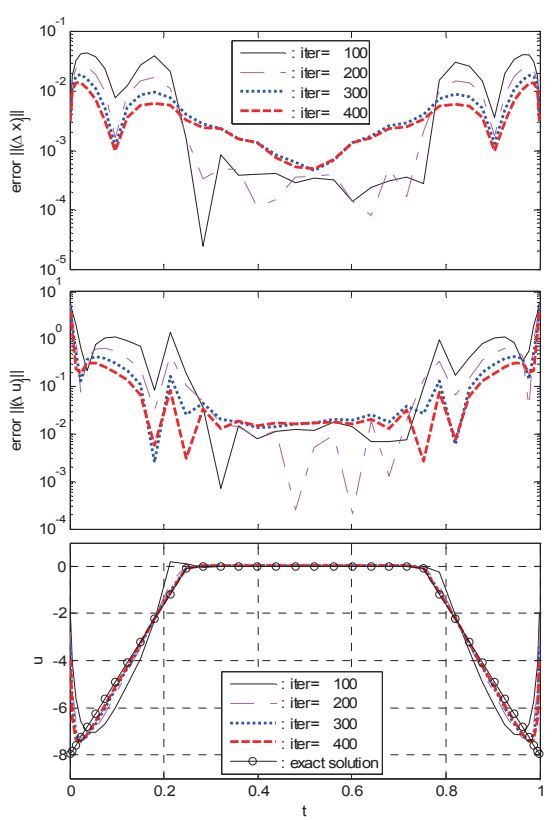

Fig. 9. Analysis results for LG points with integration method depending on number of iterations for TP2 large discrepancy with the analytical solution. For the case of TP 6, the discernible error in the control prediction exists
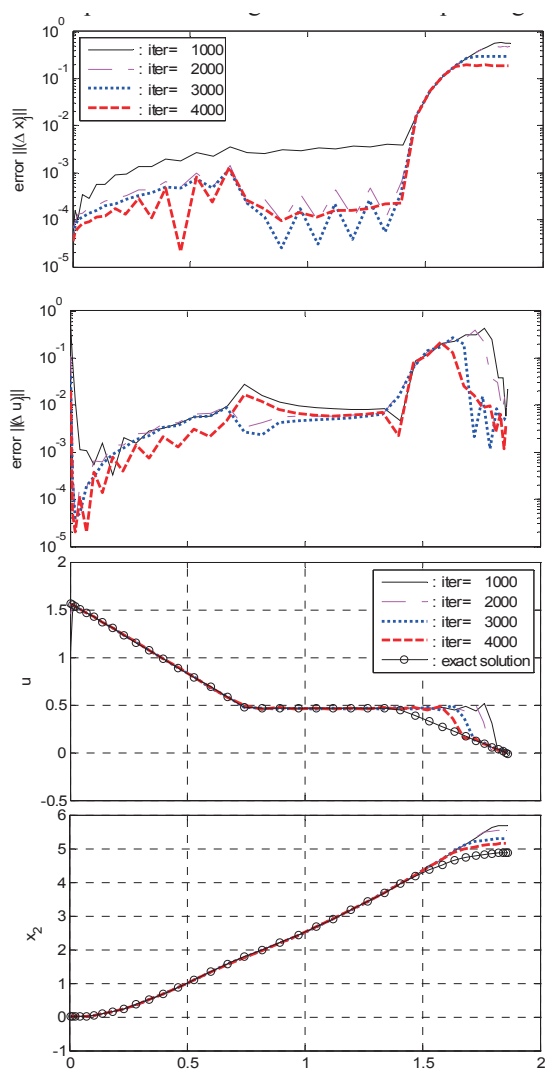

Fig. 10. Analysis results for LG points with integration method depending on number of iterations for TP5
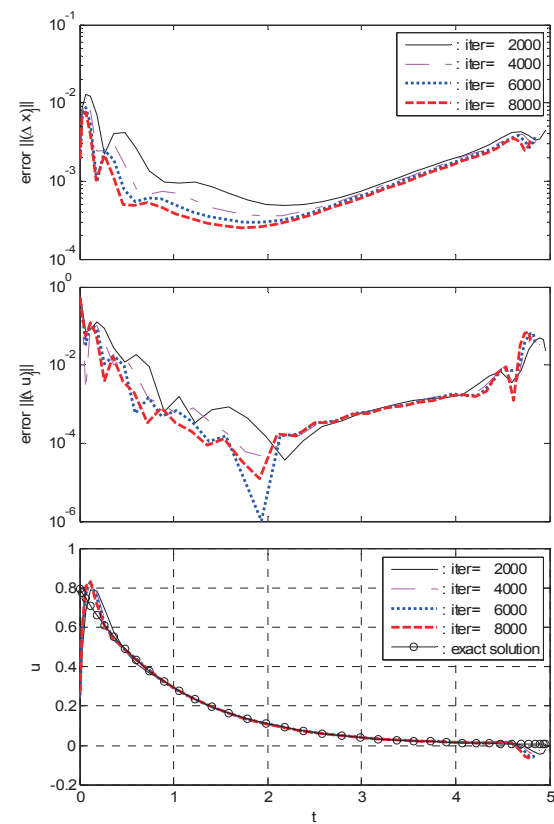

Fig. 11. Analysis results for LG points with integration method depending on number of iterations for TP6 
even after 8000 iterations, as shown in Fig.11.

Brachistochrone problem with a path constraint (TP5) are represented in Fig. 10. Both the control and the states are predicted with an extremely poor convergence rate, especially around the final end point. On the contrary, the results for the singular problem (TP6) in Fig. 11 demonstrate that the prediction accuracy of the control near both the end points presents little improvement, even after 8000 iterations. Therefore, for most case, more accurate computations are achievable only with an increased number of computational nodes at the sacrifice of a convergence rate.

Table 2 compares the number of iterations required to achive the maximum constraint violation of less than $10^{-5}$ with 40 computational nodes for each method and test problem. Nearly the same convergence can be obtained regardless of the types of collocation points. Notably, the integration method outperforms the differentiation method for TP1, TP3, TP4, and TP5.

The important findings from the comparative studies of this paper can be summarized as follows.

(i) The integration method shows faster convergence and higher accuracy than the differentiation method for most test problems, which is closely related to the condition number of the adapted Karush-KuhnTucker (KKT) system.

(ii) Also, the integration method is much less sensitive to the types of collocation points than the differentiation method which exhibits poor accuracy in the state and control solutions with LGR and LGL points as compared to those with the LG and FLGR points.

(iii) With any of LG, LGR, FLGR, and LGL points, the PS method presents extremely slow convergence around two end points.

\section{Applications To Aerospace Problems}

This section intends to present the example applications

Table 2. Comparison of the number of iterations and the maximum constraint violation $\leq 10^{-5}$

\begin{tabular}{|c|c|c|c|c|c|c|}
\hline Node & TP1 & TP2 & TP3 & TP4 & TP5 & TP6 \\
\hline \multicolumn{7}{|c|}{ integration method } \\
\hline LG & 205 & 38 & 184 & 473 & 439 & 53 \\
\hline LGR & 211 & 38 & 185 & 476 & 439 & 53 \\
\hline LGRF & 211 & 38 & 177 & 474 & 433 & 53 \\
\hline LGL & 211 & 38 & 188 & 477 & 439 & 53 \\
\hline \multicolumn{7}{|c|}{ dfferentiation method } \\
\hline LG & 269 & 38 & 243 & 497 & 493 & 53 \\
\hline LGR & 271 & 38 & 256 & 499 & 499 & 53 \\
\hline LGRF & 254 & 38 & 243 & 494 & 490 & 53 \\
\hline LGL & 280 & 38 & 255 & 496 & 498 & 53 \\
\hline
\end{tabular}

of the PS techniques to the aerospace engineering problems, which are generally large scale in their NLP formulations and are characterized by complex dynamic models. Recently, the authors performed a series of intensive studies to apply the PS methods in solving different aerospace problems. The important results closely related to this paper are summarized in this section. Firstly, the optimal takeoff trajectory of the propeller-driven airplane considering the regulatory requirements has been analyzed using the high fidelity mathematical model $[15,16]$. In addition, the optimum moon landing problems with varying altitudes of the parking orbit and the rotorcraft autorotational landing problems are investigated through $[17,18]$, respectively. In parallel with these studies, various brand-new algorithms were proposed to efficiently handle the large scale problems, to enhance computational efficiency, and to remove the limitations posed by the standard PS approaches [18-20]. All these studies utilized the important findings of the present study and the integration methods are applied using the LGL collocation points. Consequently, the main results of the studies are recapitulated to provide useful guideline for the efficient application of PS to the aerospace problems.

\subsection{Optimum take-off trajectory of a propeller-driv- en airplane.}

The NOCP that can achieve an optimum take-off trajectory of a propeller-driven airplane is formulated considering the Korean Airworthiness Standards for Very Light Airplanes (KAS-VLA). It states that the VLA should achieve a height of $15 \mathrm{~m}$ above the ground and a velocity greater than 1.3 times the stall speed $V_{S 1}$ within the landing distance of less than $500 \mathrm{~m}$. The take-off procedure consists of the ground run, rotation, and initial climb to a safe maneuvering altitude as shown in Fig. 12. Detailed NOCP formulations using the high fidelity math model and the results of the analysis with the KLA-100 airplane data can be found in [21].

The take-off trajectory is generated by assumming that the VLA has $1.1 V_{S 1}$ at the end of the take-off rotation. The NOCP to determine the landing distance $x_{f}$ is formulated using the VLA math model and the regulatoty requirements with the flap angle of 25 degrees $\left(\delta_{\text {Flap }}=25 \mathrm{deg}\right)$. Using the states and controls given in (9), the corresponding NOCP are

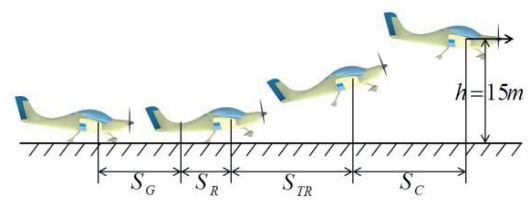

Fig. 12. Take-off procedure of VLA ([21]) 
formulated as shown in (10). The initial z-direction velocity $w\left(t_{0}\right)$ and the final longitudinal distance $x\left(t_{f}\right)$ are treated as the unknown states. Whereas, the other state variables are set to zeros at the initial time and to the trim states at the final time for three different cases of the prescribed final speed velocity $n V_{S 1}(n=1.3,1.4,1.5)$.

$$
\begin{aligned}
& \mathbf{x}=[x, y, h, u, v, w, p, q, r, \phi, \theta, \psi] \\
& \mathbf{y}=[p, q, r, \phi, \theta, \psi] \\
& \mathbf{u}=\left[\delta_{A}, \delta_{E}, \delta_{R}, \delta_{T}\right] \\
& \min J=x_{f}+t_{f}+\frac{1}{2} \int_{t_{0}}^{t_{f}}\left(\mathbf{y}^{T} \mathbf{y}+5 \mathbf{u}^{T} \mathbf{u}\right) d t \\
& \text { subject to } \dot{\mathbf{x}}=\mathbf{f}(\mathbf{x}, \mathbf{u}), t \in\left[t_{0}, t_{f}\right] \\
& u\left(t_{0}\right)=1.1 V_{S 1} \\
& h\left(t_{f}\right)=15.0 \mathrm{~m}
\end{aligned}
$$

The formulated NOCPs are solved using the integration method and the LGL nodes. Fig. 13 represents the variation of states and controls during the take-off procedure. Table 3 summarizes the estimated flight times and final distances with the different final flight speeds, which proves that the

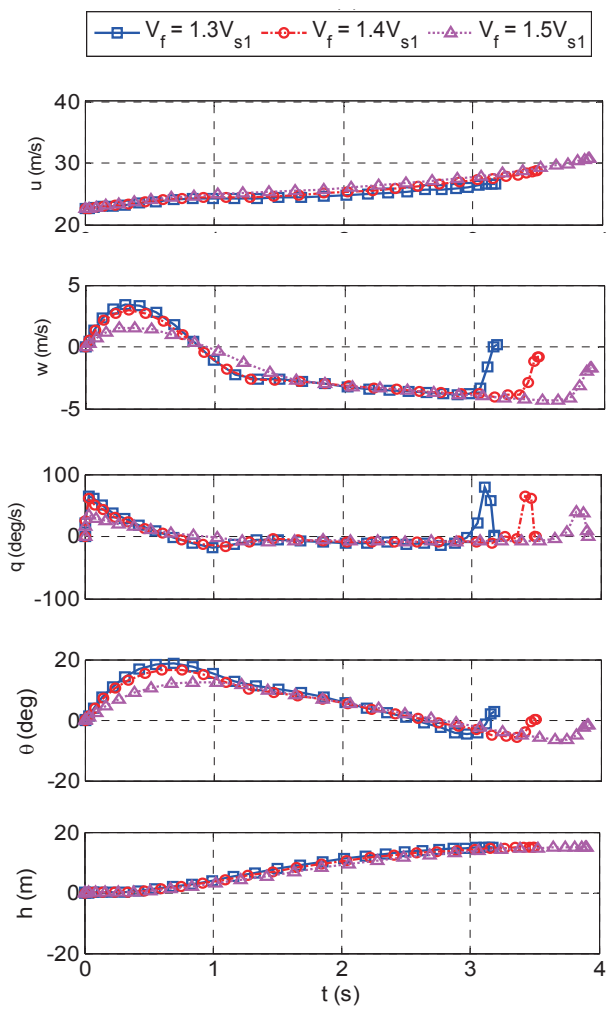

Fig. 13. Optimal take-off trajectory and controls

Table 3. Computed flight-time and take-off distance Final flight speed

\begin{tabular}{|l|c|c|c|}
\hline Final flight speed & $1.3 V_{s 1}$ & $1.4 V_{s 1}$ & $1.5 V_{s 1}$ \\
\hline Flight time (sec) & 3.17 & 3.49 & 3.90 \\
\hline Final distance $(\mathrm{m})$ & 77 & 88 & 102 \\
\hline
\end{tabular}

KLA-100 can meet the KAS-VLA regulations for the take-off procedure.

\subsection{Optimum lunar landing trajectory of the space- craft.}

The optimum trajectories of the spacecraft are predicted for the soft lunar landing from the Moon's parking orbit as shown in Fig. 14. The effects of the initial altitude of the parking orbit on the minimum fuel consumption are investigated. The objective function is defined using the consumed fuel mass and the final landing conditions are imposed as the constraints. The corresponding NOCP can be formulated with the spacecraft mass $m(t)$, position $\mathrm{r}(t)$, and thrust vector $\mathrm{T}(t)$ as:

$\min J(\mathbf{r}, \mathbf{T}, t)=m\left(t_{0}\right)-m\left(t_{f}\right)$

Subject to

$$
\begin{aligned}
& \ddot{\mathbf{r}}=-\frac{\mu}{r^{3}} \mathbf{r}+\frac{\mathbf{T}}{m} \\
& \dot{m}=-\frac{\|\mathbf{T}\|}{I_{s p} g_{0}} \\
& 0.0 \leq v_{r}\left(t_{f}\right) \leq 1 \quad(\mathrm{~m} / \mathrm{sec}) \\
& -0.01 \leq v_{\theta}\left(t_{f}\right), v_{h}\left(t_{f}\right) \leq 0.01
\end{aligned}
$$

where

$$
\begin{aligned}
& v_{r}=\mathbf{e}_{r} \cdot \dot{\mathbf{r}} \quad \mathbf{e}_{r}=\mathbf{r} / r \\
& v_{h}=\mathbf{e}_{h} \cdot \dot{\mathbf{r}} \quad, \quad \mathbf{e}_{h}=(\mathbf{r} \times \dot{\mathbf{r}}) /\|\mathbf{r} \times \dot{\mathbf{r}}\| \\
& v_{\theta}=\mathbf{e}_{\theta} \cdot \dot{\mathbf{r}} \quad \mathbf{e}_{\theta}=\mathbf{e}_{h} \times \mathbf{e}_{r}
\end{aligned}
$$

The main parameters for the spacecraft math model are listed in Table 4.

Figure 15 shows the optimum trajectory and the variations in the spacecraft mass and thrust, which shows the fuel consumptions for each trajectory present minor variations with different initial altitudes. Therefore, the mission time and mission scenario of the spacecraft can be

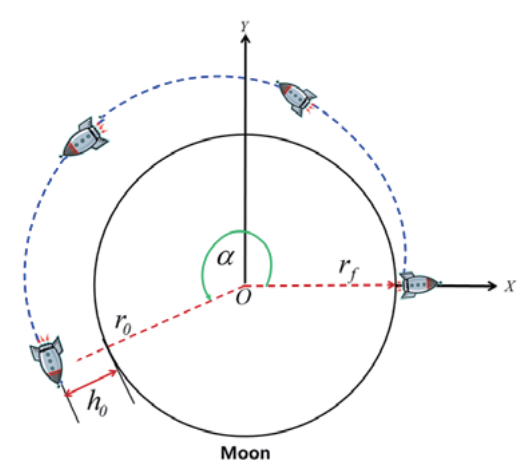

Fig. 14. Schematic diagram for soft lunar landing [17] 
main factors in determining the initial altitude for the entry of the landing.

Table 4. Parameters for lunar landing problem

\begin{tabular}{|c|l|}
\hline$r_{0}=1748.0 \sim 1838.0 \mathrm{~km}$ & $I_{s p}=316.9 / \mathrm{sec}$ \\
$T_{\max }=1.7 \mathrm{~kg} \mathrm{~km} / \mathrm{sec}^{2}$ & $r_{m}=1738.0 \mathrm{~km}$ \\
$g_{0}=0.0098 \mathrm{~km} / \mathrm{sec}^{2}$ & $m_{0}=600 \mathrm{~kg}$ \\
$\mu=4902.7779 \mathrm{~km} / \mathrm{sec}^{2}$ & $h_{0}=r_{0}-r_{m}$ \\
\hline
\end{tabular}
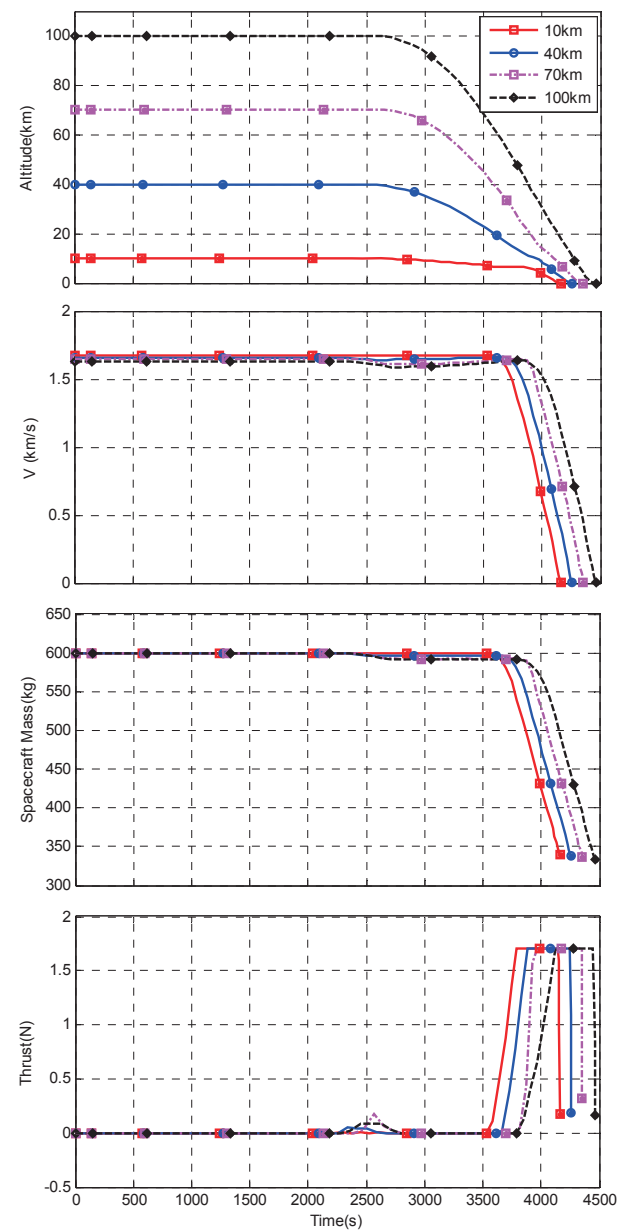

Fig. 15. Variations of height, mass, and thrust

\subsection{Optimal autorotation trajectory of the rotorcraft}

This study intends to use a high-fidelity rotorcraft model, which typically includes fast rotor dynamics, in the NOCP analyses to accurately predict the emergency procedures of the rotorcraft after the engine failure. For this purpose, the numerical time-scale separation techniques (NTSS) are proposed to separate the fast dynamics from the slow states of the rigid body during the NLP solution process [18]. Also, the applications of the moving horizon approach and the residualization method are proposed to guarantee solution convergence and to preserve the sensitivity of the cost and constraint functions to the fast states. The Helicopter Trim, Linearization and Simulation program [22-23] (HETLAS ) are used to model the BO105 helicopter. The flap, lead-lag, and RPM dynamics are selected for the blade motions in building the dynamic model. Therefore, the helicopter, states and controls can be defined as follows:

$$
\begin{aligned}
\dot{\mathbf{x}} & =\mathbf{f}\left(\mathbf{x}_{R}, \mathbf{x}_{F}, \mathbf{x}_{I}, \mathbf{u}, t\right)=\left(\mathbf{f}_{R}, \mathbf{f}_{F}, \mathbf{f}_{I}\right)^{T} \\
\mathbf{x} & =\left(\mathbf{x}_{R}, \mathbf{x}_{F}, \mathbf{x}_{I}, \mathbf{x}_{\Omega}\right)^{T} \\
\mathbf{u} & =\left(\theta_{0}, \theta_{1 C}, \theta_{1 S}, \theta_{T R}\right)^{T} \\
\mathbf{x}_{R} & =(u, v, w, p, q, r, \phi, \theta, \psi, x, y, h)^{T} \\
\mathbf{x}_{F} & =\left(\dot{\beta}_{0}, \dot{\beta}_{1 C}, \dot{\beta}_{1 S}, \dot{\beta}_{1}, \beta_{0}, \beta_{1 C}, \beta_{1 S}, \beta_{1}\right)^{T} \\
\mathbf{x}_{I} & =\left(\lambda_{0}, \lambda_{C}, \lambda_{1 S}\right)^{T} \\
\mathbf{x}_{\Omega} & =(\Omega)
\end{aligned}
$$

where $\mathbf{X}_{R}, \mathbf{X}_{F}, \mathbf{X}_{I}$ and $\mathbf{X}_{\Omega}$ represent the rigid body, flap $(\beta)$, inflow $(\lambda)$ and RPM states, respectively. As the results of the high-fidelity math modeling, the system dynamics include 24 states and four controls. Furthermore, the states for $\left(\mathbf{X}_{F}\right.$ $\mathbf{X}_{I}, \mathbf{X}_{\Omega}$ ), which are not directly used for the control purposes, show relatively fast responses compared to those for the rigid body states $\mathbf{X}_{R}$, which make the NOCP applications nearly impossible with the traditional PS approaches.

Ref. 18 proposed the numerical time-scale separation techniques (NTSS) combined with a moving horizon approach for efficient time integration of the fast dynamics.

Table 5. Comparison of system size and CPU time with a different number of segments for fast dynamics [18]

\begin{tabular}{|c|c|c|c|c|}
\hline \multirow{2}{*}{ Problem size and computing time } & \multicolumn{4}{|c|}{ Number of time segments for fast dynamics } \\
\cline { 2 - 5 } & 60 & 80 & 100 & 120 \\
\hline Number of NLP design variables & \multicolumn{4}{|c|}{2041 (120 nodes) } \\
\hline Number of NLP constraints & \multicolumn{4}{|c|}{2654 (120 nodes) } \\
\hline Number of fast dynamic constraints & 6480 & 8640 & 10800 & 12960 \\
\hline Average CPU time per iteration(sec) & 33.5 & 37.5 & 38.4 & 42.06 \\
\hline
\end{tabular}


This technique only allows us to define the design variables in the NLP formulation with the slow states. Therefore, it can greatly reduce the system size and is extremely efficient in solving the large scale aerospace problem with separable time scales. Table 5 compares the size of the NLP and the CPU time required for NOCP analyses for the autorotational landing problem of the $\mathrm{BO}-105$ helicopter. It shows that the NTSS techniques incur minor increases in the CPU time even though the number of dynamic constraints for the fast dynamics double. Therefore, the NTSS techniques can effectively remove the limitations imposed by the traditional PS approaches.

The results of the example applications of the PS method to the aerospace engineering problems show that the PS methods are efficiently applicable in analyzing the complex and large-scale aerospace problems. In addition, the selection of the best combination among the collocation points and the trascription methods can be cruicial as investigated through this paper.

\section{Conclusion}

This paper focuses on investigating the pros and cons of various transciption techniques for the Legendre PS method. Comparative studies were conducted using six carefully seclected NOCP test problems to identify the best combination of collocation point types and of time integration methods. The Legendre PS method exhibits a strong convergence with the present initial approximation of the states and the control. For most test problems, the integration method outperformed the differentiation method with a better convergence rate and higher accuracy. The analyses using the differentiation method had a stronger dependency on the type of collocation points than those using the integration method. The LG and FLGR points generally provided better prediction accuracy than the LGR and LGL points for the differentiation method. The integration method provided nearly the same level of accuracy regardless of the type of collocation points.

The analyses for the NOCPs with path constraints as well as for a singular problem demonstrated extremely poor prediction accuracy and a slow convergence rate with the given number of computational nodes around two end points. In these cases, a trade-off between prediction accuracy and convergence rate with an increase in the number of nodes is the best solution. In a case when the accurate estimation of the states and controls around the initial points is required as in the model predictive control, the PS method with slow convegence in predicting the intial controls can provide unstable divergent control inputs to the system. Although the study of the costate estimations will be a topic of a future study, the results of the present study of various types of test problems can be used for the best selection of transcription techniques with the PS method. Finally, the applications of the PS methods to the trajectory optimization for the aircaft and spacecraft were demonstrated and the results denote that the PS method can be a useful tool in solving complex aerospace engineering problems.

\section{Acknowledgements}

This work was support by the Faculty Research Fund of Konkuk University in 2012.

\section{APPENDIX A. Quadrature weights, integra- tion and differentiation matrices for the Leg- endre PS methods}

Table A1 shows formulas to compute the collocation points for each PS method and Table A2 represents the corresponding quadrature weights $\left\{w_{k}\right\}_{k=0}^{k=K}[5,6]$. The computational nodes and weights for the NOCP analysis are defined in Table A3. The differentiation and integration matrices can be built by using the following formulas:

Table A1. Polynomial equations to get quadrature points

\begin{tabular}{|l|l|l|}
\hline types & $\begin{array}{c}\text { roots of } \\
g(\tau)=0\end{array}$ & polynomial $g(\tau)$ \\
\hline LG & $\left\{\tau_{k}^{L G}\right\}_{k=0}^{k=K}$ & $g(\tau)=L_{K+1}(\tau) / k_{K+1}$ \\
\hline LGR & $\left\{\tau_{k}^{L G R}\right\}_{k=0}^{k=K}$ & $g(\tau)=\left\{L_{K+1}(\tau)+L_{K}(\tau)\right\} / k_{K+1}$ \\
\hline FLGR & $\left\{\tau_{k}^{F L R}\right\}_{k=0}^{k=K}$ & $g(\tau)=\left\{L_{K+1}(\tau)-L_{K}(\tau)\right\} / k_{K+1}$ \\
\hline LGL & $\left\{\tau_{k}^{L G L}\right\}_{k=0}^{k=K}$ & $\left.g(\tau)=\left\{L_{K+1}(\tau)-L_{K-1}(\tau)\right\}\right) / k_{K+1}$ \\
\hline
\end{tabular}

Table A2. Quadrature weights at $k$-th quadrature point

\begin{tabular}{|l|l|}
\hline types & weights \\
\hline LG & $w_{k}=\frac{2}{1-\left(\tau_{k}^{L G}\right)^{2}} \times \frac{1}{\left[L_{K+1}\left(\tau_{k}^{G R}\right)\right]^{2}}$ \\
\hline LGR & $w_{k}=\frac{1-\tau_{k}^{L G R}}{(K+1)^{2}\left[L_{K}\left(\tau_{k}^{L G R}\right)\right]^{2}}$ \\
\hline FLGR & $w_{k}=\frac{1+\tau_{k}^{F L G R}}{(K+1)^{2}\left[L_{K}\left(\tau_{k}^{F L G R}\right)\right]^{2}}$ \\
\hline LGL & $w_{k}=\frac{2}{K(K+1)\left\{L_{K}\left(\tau_{k}^{L G L}\right)\right\}^{2}}$ \\
\hline
\end{tabular}


Table A3. Nodes and weights in the computational domain

\begin{tabular}{|l|ll|}
\hline types & Rearranged set of nodes and weights \\
\hline LG & $\begin{array}{l}\left\{\tau_{j}\right\}_{j=0}^{N}=\{-1\}+\left\{\tau_{k}^{L G}\right\}_{k=0}^{N-2}+\{1\}, \quad K=N-2 \\
\left\{w_{j}\right\}_{j=0}^{N}=\{0\}+\left\{w_{k}\right\}_{k=0}^{N-2}+\{0\}\end{array}$ \\
\hline LGR & $\begin{array}{l}\left\{\tau_{j}\right\}_{j=0}^{N}=\left\{\tau_{k}^{L G R}\right\}_{k=0}^{N-1}+\{1\}, \\
\left\{w_{j}\right\}_{j=0}^{N}=\left\{w_{k}\right\}_{k=0}^{N-1}+\{0\}\end{array}$ & \\
\hline FLGR & $\begin{array}{l}\left\{\tau_{j}\right\}_{j=0}^{N}=\{-1\}+\left\{\tau_{k}^{F L G R}\right\}_{k=0}^{N-1}, \\
\left\{w_{j}\right\}_{j=0}^{N}=\{0\}+\left\{w_{k}\right\}_{k=0}^{N-1}\end{array}$ & $K=N-1$ \\
\hline LGL & $\begin{array}{l}\left\{\tau_{j}\right\}_{j=0}^{N}=\left\{\tau_{k}^{L G L}\right\}_{k=0}^{N}, \\
\left\{w_{j}\right\}_{j=0}^{N}=\left\{w_{k}\right\}_{k=0}^{N}\end{array}$ \\
\hline
\end{tabular}

$D_{j k}=\dot{\phi}_{k}\left(\tau_{j}\right)= \begin{cases}\frac{\dot{g}\left(\tau_{j}\right)}{\left(\tau_{j}-\tau_{k}\right) \dot{g}\left(\tau_{k}\right)}, & (j \neq k) \\ \frac{\ddot{g}\left(\tau_{k}\right)}{2 \dot{g}\left(\tau_{k}\right)}, & (j=k)\end{cases}$

$I_{j k}=\frac{1}{\dot{g}\left(\tau_{k}\right)} \int_{\tau_{0}}^{\tau_{j}} \frac{g(\tau)}{\left(\tau-\tau_{k}\right)} d \tau$

Remark: $k_{K+1}$ is the leading coefficient of the $(K+1)^{\text {th }}$ order Legendre polynomial $L_{K+1}(\tau)$

\section{APPENDIX B. NOCP Test Problems}

[TP1] Minimum energy problem with bounded control [7] $\min J=\int_{0}^{t_{f}} u d t$

subject to

$$
\left(\begin{array}{l}
\dot{x}_{1} \\
\dot{x}_{2}
\end{array}\right)=\left(\begin{array}{l}
x_{2} \\
u-g
\end{array}\right),\left(\begin{array}{l}
x_{1,0} \\
x_{2,0}
\end{array}\right)=\left(\begin{array}{c}
10 \\
-2
\end{array}\right),\left(\begin{array}{l}
x_{1, f} \\
x_{2, f}
\end{array}\right)=\left(\begin{array}{l}
0 \\
0
\end{array}\right), \begin{aligned}
& 0 \leq u \leq 3 \\
& g=1.2
\end{aligned}
$$

The optimal solution is

$t_{f}^{*}=\left\{2 x_{2,0}+4 \sqrt{0.5 x_{2,0}^{2}+1.5 x_{1,0}}\right\} / 3, \quad t_{s}^{*}=0.5 t_{f}^{*}+x_{2,0} / 3$

$J^{*}=3\left(t_{f}^{*}-t_{s}^{*}\right)$

(i) $0 \leq t \leq t_{s}^{*}$

$$
\left(x_{1}^{*}, x_{2}^{*}, u^{*}\right)=\left(-0.75 t^{2}+x_{2,0} t+x_{1,0},-1.5 t+x_{2,0}, 0\right)
$$

(ii) $t_{s}^{*} \leq t \leq t_{f}^{*}$ with $\alpha=x_{2,0}-3 t_{s}^{*}$

$$
\left(x_{1}^{*}, x_{2}^{*}, u^{*}\right)=\left(\left\{0.75 t^{2}+\alpha t+1.5\left(t_{s}^{*}\right)^{2}+x_{1,0}\right\} 1.5 t+\alpha, 3\right)
$$

[TP2] Minimum energy problem with path constraints [10]

$$
\min J=\int_{0}^{1} u^{2} d t
$$

subject to

$$
\left(\begin{array}{l}
\dot{x}_{1} \\
\dot{x}_{2}
\end{array}\right)=\left(\begin{array}{l}
x_{2} \\
u
\end{array}\right),\left(\begin{array}{l}
x_{1,0} \\
x_{2,0}
\end{array}\right)=\left(\begin{array}{l}
0 \\
1
\end{array}\right),\left(\begin{array}{l}
x_{1, f} \\
x_{2, f}
\end{array}\right)=\left(\begin{array}{l}
0 \\
-1
\end{array}\right), \begin{aligned}
& x_{1}(t) \leq l \\
& l=1 / 12
\end{aligned}
$$

The optimal solution is

$J^{*}=16 / 3$

(i) $0 \leq t \leq 3 l$

$$
\left(x_{1}^{*}, x_{2}^{*}, u^{*}\right)=\left(l\left\{1-\left(1-\frac{t}{3 l}\right)^{3}\right\},\left(1-\frac{t}{3 l}\right)^{2},-\frac{2}{3 l}\left(1-\frac{t}{3 l}\right)\right)
$$

(ii) $3 l \leq t \leq 1-3 l$

$$
\left(x_{1}^{*}, x_{2}^{*}, u^{*}\right)=(l, 0,0)
$$

(iii) $1-3 l \leq t \leq 1$ with $\alpha(t)=1-(1-t) / 3 l$

$$
\left(x_{1}^{*}, x_{2}^{*}, u^{*}\right)=\left(l\left\{1-\alpha(t)^{3}\right\},-\{1-\alpha(t)\}^{2},-\frac{2}{3 l}\{1-\alpha(t)\}\right)
$$

[TP3] Minimum time problem with bounded control [11] $\min J=t_{f}$

subject to

$\left(\begin{array}{l}\dot{x}_{1} \\ \dot{x}_{2}\end{array}\right)=\left(\begin{array}{l}x_{2} \\ u\end{array}\right),\left(\begin{array}{l}x_{1,0} \\ x_{2,0}\end{array}\right)=\left(\begin{array}{l}3 \\ 2\end{array}\right),\left(\begin{array}{l}x_{1, f} \\ x_{2, f}\end{array}\right)=\left(\begin{array}{l}0 \\ 0\end{array}\right),|u| \leq 1$

The optimal solution is

$$
\begin{aligned}
& t_{s}^{*}=2+\sqrt{5}, \quad t_{f}^{*}=2(1+\sqrt{5}) \\
& \text { (i) } 0 \leq t \leq t_{s}^{*}, \quad\left(x_{1}^{*}, x_{2}^{*}, u^{*}\right)=\left(-0.5 t^{2}+2 t+3,-t+2,-1\right) \\
& \text { (ii) } t_{s}^{*} \leq t \leq t_{f}^{*}, \quad\left(x_{1}^{*}, x_{2}^{*}, u^{*}\right)=\left(0.5 t^{2}-t_{f}^{*} t+0.5\left(t_{f}^{*}\right)^{2}, t-t_{f}^{*}, 1\right)
\end{aligned}
$$

[TP4] Brachistochrone problem without constraints [10] $\min J=t_{f}$

subject to

$$
\left(\begin{array}{l}
\dot{x}_{1} \\
\dot{x}_{2}
\end{array}\right)=\left(\begin{array}{l}
\sqrt{2 g x_{2}} \cos u \\
\sqrt{2 g x_{2}} \sin u
\end{array}\right),\left(\begin{array}{l}
x_{1,0} \\
x_{2,0}
\end{array}\right)=\left(\begin{array}{l}
0 \\
0
\end{array}\right),\left(x_{1, f}\right)=(L)
$$

The optimal solution with $\beta(t)=\sin 2 \omega t$ is

$$
\begin{aligned}
& \left(x_{1}^{*}, x_{2}^{*}, u^{*}\right)=\left(\frac{2 L}{\pi}(\omega t-0.5 \beta(t)), \frac{2 L}{\pi} \beta(t)^{2}, \frac{1}{2} \pi-\omega t\right) \\
& t_{f}^{*}=\sqrt{\pi L / g}
\end{aligned}
$$

[TP5] Brachistochrone problem with path constraints [10] $\min J=t_{f}$

subject to

$$
\left(\begin{array}{l}
\dot{x}_{1} \\
\dot{x}_{2}
\end{array}\right)=\left(\begin{array}{l}
\sqrt{2 g x_{2}} \cos u \\
\sqrt{2 g x_{2}} \sin u
\end{array}\right),\left(\begin{array}{l}
x_{1,0} \\
x_{2,0}
\end{array}\right)=\left(\begin{array}{l}
0 \\
0
\end{array}\right),\left(x_{1, f}\right)=(L)
$$


$x_{2} \leq x_{1} \tan \alpha+h$ with prescribed $\alpha$ and $h$

The optimal solution is

$$
\begin{aligned}
\omega_{1} & =\sqrt{g(\alpha-0.5 \pi+\cot \alpha) /(2 h \cot \alpha)} \\
\omega_{2} & =\sqrt{g(\alpha+\cot \alpha)(2 L+h \cot \alpha)} \\
t_{s 1}^{*} & =(0.5 \pi-\alpha) / \omega_{1} \\
t_{s 2}^{*} & =t_{f}-\alpha / \omega_{2} \\
t_{f}^{*} & =\sqrt{2(L+h \cot \alpha)(\alpha+\cot \alpha) / g} \\
& -\sqrt{2 h \cot \alpha(\alpha-0.5 \pi+\cot \alpha) / g}
\end{aligned}
$$

(i) $0 \leq t \leq t_{s 1}^{*}$

$$
\left(\begin{array}{l}
x_{1}^{*} \\
x_{2}^{*} \\
u^{*}
\end{array}\right)=\left(\begin{array}{c}
0.5 g\left\{t-0.5 \sin \left(2 \omega_{1} t\right) / \omega_{1}\right\} / \omega_{1} \\
0.5 g \sin ^{2}\left(\omega_{1} t\right) / \omega_{1}^{2} \\
0.5 \pi-\omega_{1} t
\end{array}\right)
$$

(ii) $t_{s 1}^{*}<t \leq t_{s 2}^{*}$

$$
\begin{aligned}
& x_{1}^{*}(t)=\left\{\begin{array}{l}
x_{1}^{*}\left(t_{s 1}^{*}\right)+\sqrt{2 g x_{2}^{*}\left(t_{s 1}^{*}\right)}\left(t-t_{s 1}^{*}\right) \cos \alpha \\
+0.25 g\left(t-t_{s 1}\right)^{2} \cos \alpha \sin \alpha
\end{array}\right\} \\
& x_{2}^{*}(t)=\left\{\sqrt{x_{2}^{*}\left(t_{s 1}^{*}\right)}+0.5 \sqrt{2 g}\left(t-t_{s 1}^{*}\right) \sin \alpha\right\}^{\prime} \\
& u^{*}(t)=\alpha
\end{aligned}
$$

(iii) $t_{s 2}^{*}<t \leq t_{f}^{*}$

$$
\begin{aligned}
x_{1}^{*}(t)= & x_{1}^{*}\left(t_{s 2}^{*}\right)+\left(\omega_{2} \sqrt{2 g x_{2}^{*}\left(t_{s 2}^{*}\right)}-g \cos \omega_{2}\left(t_{s 2}-t_{f}\right)\right) \\
& \times\left\{\sin \omega_{2}\left(t-t_{f}\right)-\sin \omega_{2}\left(t_{s 2}-t_{f}\right)\right\} / \omega_{2}^{2} \\
& +0.5 g\left(\begin{array}{l}
\omega_{2} t+0.5 \sin 2 \omega_{2}\left(t-t_{f}\right) \\
-t_{s 2}+0.5 \sin 2 \omega_{2}\left(t_{s 2}-t_{f}\right)
\end{array}\right) / \omega_{2}^{2} \\
x_{2}^{*}(t) & =\left\{\sqrt{x_{2}^{*}\left(t_{s 2}^{*}\right)}+0.5 \sqrt{2 g}\left\{\begin{array}{l}
\cos \omega_{2}\left(t-t_{f}\right) \\
-\cos \omega_{2}\left(t_{s 2}-t_{f}\right)
\end{array}\right\} / \omega_{2}\right\} \\
u^{*}(t) & =\omega_{2}\left(t_{f}^{*}-t\right)
\end{aligned}
$$

[TP6] Singular problem with bounded control [12]

$$
\min J=\frac{1}{2} \int_{0}^{t_{f}} x_{1}^{2}+x_{2}^{2} d t
$$

subject to

$$
\left(\begin{array}{l}
\dot{x}_{1} \\
\dot{x}_{2}
\end{array}\right)=\left(\begin{array}{l}
x_{2} \\
u
\end{array}\right),\left(\begin{array}{l}
x_{1,0} \\
x_{2,0}
\end{array}\right)=\left(\begin{array}{c}
0.8 \\
-0.8
\end{array}\right),\left(\begin{array}{l}
x_{1, f} \\
x_{2, f}
\end{array}\right)=\left(\begin{array}{c}
0.01 \\
-0.01
\end{array}\right),|u| \leq 1
$$

The optimal solution is

$$
\left(x_{1}^{*}, x_{2}^{*}, u^{*}\right)=\left(0.8 e^{-t},-0.8 e^{-t}, 0.8 e^{-t}\right), \quad t_{f}^{*}=\ln 80
$$

\section{References}

[1] Kim, C.-J., Sung, S., Park, S. H. and Jung, S.-N., "Selection of Rotorcraft Models for Application to Optimal Control Problems," Journal of Guidance, Control, and Dynamics, Vol. 31, No. 5, 2008, pp. 1386 1399.

[2] Kim, C.-J., Lee, J., Byun, Y. H. and Yu, Y. H., "Nonlinear optimal control analysis of helicopter maneuver problems using the indirect method," Transactions of the Japan Society for Aeronautical and Space Sciences, Vol. 51, No. 171, 2008, pp. 43-51.

[3] Kim, C.-J., Sung, S., Yang, C. D. and Yu, Y. H., "Rotorcraft Trajectory Tracking Using the Sate-Dependent Riccati Equation Controller," Transactions of the Japan Society for Aeronautical and Space Sciences, Vol. 51, No. 173, 2008, pp. 184-192.

[4] Kim, C.-J., Lee, J. H., Park, S. H., Sung, S. and Jung, S.-N., "Nonlinear Optimal Control Analysis Using State-Dependent Matrix Exponential and Its Integrals," Journal of Guidance, Control, and Dynamics, Vol. 31, No. 5, pp. 1386-1399, 2008.

[5] Benson, D., A Gauss Pseudospectral Transcription for Optimal Control, Ph.D. Thesis, MIT, Department of Aeronautics and Astronautics, November 2004.

[6] Huntington, G. T., Advancement and Analysis of a Gauss Pseudospectral Transcription for Optimal Control Problems, Ph.D. Thesis, MIT, Department of Aeronautics and Astronautics, June 2007

[7] Darby, C. L., HP-Pseudo-spectral Method for Solving Continuous-Time Nonlinear Optimal Control Problems, Ph. D. Thesis, University of Florida, May 2011.

[8] D. Garg, M. Patterson, W. W. Hager, A. V. Rao, D. A. Benson, and G. T. Huntington, "A Unified Framework for the Numerical Solution of Optimal Control Problems Using Pseudospectral methods," Automatica, Published Online, August 2010. DOI:10.1016/j.automatica.2010.06.048.

[9] Williams, P., "A Gauss-Lobatto Quadrature Method for Solving Optimal Control Problems," Australian and New Zealand Industrial and Applied Mathematics Journal, Vol. 47, 2005, pp. 101-115.

[10] Bryson, A. E., Jr. and Ho, Y. C., Applied Optimal Control, Hemisphere Publishing, 1975.

[11] Singh, B., A Weighted Residual Framework for Formulation and Analysis of Direct Transcription Methods for Optimal Control, Ph.D. Thesis, Texas A\&M University, December 2010.

[12] Kirk, D. E., Optimal Control Theory; An Introduction, Dover, New York, 1970.

[13] Fabien, B. C., "Implementation of a robust SQP algorithm," Optimization Method \& Software, Vol. 23, No. 6 , October 2008, pp. 827-846.

[14] Kim, C.-J., Sung, S. and Shin, K., "Pseudo-spectral Application to Nonlinear Optimal Trajectory Generation of a Rotorcraft," The First International Conference on Engineering and Technology Innovation, Kenting, Taiwan, November 1115,2011

[15] Kim, C.-J. et al., "Flight Dynamics Analyses of a Propeller-Driven Airplane (I): Aerodynamic and Inertial Modeling of the Propeller," IJASS, Vol. 15, No. 4, pp. 345-355, 2014. 
[16] Kim, C.-J. et al., "Flight Dynamics Analyses of a Propeller-Driven Airplane (II): Building a High-Fidelity Mathematical Model and Applications," IJASS, Vol. 15, No. 4, pp. 356-365, 2014.

[17] Lee, D. H., Hur, S. U. and Kim, C.-J., "Study on Optimum Lunar Landing Trajectories Considering Initial Altitude of Lunar Parking Orbit," KSASS Autumn Conference, Jeju, Korea, November, 2014.

[18] Kim, C.-J., Sung, S., Park, S. H., Jung, S. N. and Park, T. S., "Numerical Time Scale Separation for Nonlinear Optimal Control Analyses with Applications to Rotorcrafts," AIAA Journal of Guidance, Control and Dynamics, Vol. 37, No. 2, March-April 2014, pp. 658-673.

[19] Kim, C.-J., Park, S. H., S.-N. Jung, and Sung, S., "Generalized Computational Nodes for Pseudospectral Methods," IJASS, Vol. 15, No. 2, 2014, pp. 183-189.

[20] Kim, C.-J. and Sung, S., "Efficient ST Techniques for
Nonlinear Optimal Control Analyses Using a Pseudospectral Framework," IEEE Transactions on Control Systems Technology, Vol. 23, No. 3, May 2015, pp. 1110-1116.

[21] Park, T. S., Research on Dynamic Modeling, Flight Dynamic Analysis and Optimum Trajectory Generation, M.S. Thesis, Department of Aerospace Information Systems Engineering, Konkuk University, Seoul, Korea, December, 2013.

[22] Yun, Y.-H., Kim, C.-J., Shin, K.-C., Yang, C.-D. and Cho, I.-J., "Building the Flight Dynamic Analysis Program, HETLAS, for the Development of Helicopter FBW System," 1st Asian Australian Rotorcraft Forum and Exhibition 2012, Bexco, Busan, Korea, February 12-15, 2012.

[23] Kim, C.-J., Shin, K.-C., Yang, C.-D. and Cho, I.-J., "Interface Features of Flight Dynamic Analysis Program, HETLAS, for the Development of Helicopter FBW System," 1st Asian Australian Rotorcraft Forum and Exhibition 2012, Bexco, Busan, Korea, February 12-15, 2012. 